\title{
Effect of mechanical loading on the metabolic activity of cells in the temporomandibular joint: a systematic review
}

\author{
Beatriz F. Betti ${ }^{1,2,4}$ (D) Vincent Everts ${ }^{2} \cdot$ Johannes C. F. Ket $^{3} \cdot$ Hessam Tabeian $^{2}$ • \\ Astrid D. Bakker ${ }^{2}$ • Geerling E. Langenbach ${ }^{2}$ - Frank Lobbezoo 4
}

Received: 20 December 2016 / Accepted: 21 July 2017 /Published online: 1 August 2017

(C) The Author(s) 2017. This article is an open access publication

\begin{abstract}
Objectives The purpose of this systematic review was to elucidate how different modalities and intensities of mechanical loading affect the metabolic activity of cells within the fibrocartilage of the temporomandibular joint (TMJ).

Materials and methods A systematic review was conducted according to PRISMA guidelines using PubMed, Embase, and Web of Science databases. The articles were selected following a priori formulated inclusion criteria (viz., in vivo and in vitro studies, mechanical loading experiments on TMJ, and the response of the TMJ).

A total of 254 records were identified. After removal of duplicates, 234 records were screened by assessing eligibility criteria for inclusion. Forty-nine articles were selected for full-
\end{abstract}

Electronic supplementary material The online version of this article (doi:10.1007/s00784-017-2189-9) contains supplementary material, which is available to authorized users.
Beatriz F. Betti
b.f.betti@acta.nl
Vincent Everts
v.everts@acta.nl
Johannes C. F. Ket
jcf.ket@vu.nl
Hessam Tabeian
h.tabeian@acta.nl
Astrid D. Bakker
a.bakker@acta.nl
Geerling E. Langenbach
g.langenbach@acta.nl

text assessment. Of those, 23 were excluded because they presented high risk of bias or were reviews. Twenty-six experimental studies were included in this systematic review: 15 in vivo studies and 11 in vitro ones.

Conclusion The studies showed that dynamic mechanical loading is an important stimulus for mandibular growth and for the homeostasis of TMJ cartilage. When this loading is applied at a low intensity, it prevents breakdown of inflamed cartilage. Yet, frequent overloading at excessive levels induces accelerated cell death and an increased cartilage degradation.

Clinical Significance Knowledge about the way temporomandibular joint (TMJ) fibrocartilage responds to different types and intensities of mechanical loading is important to
Frank Lobbezoo

f.lobbezoo@acta.nl

1 Department of Orthodontics, Academic Centre for Dentistry Amsterdam, University of Amsterdam and VU University, Amsterdam, The Netherlands

2 Department of Oral Cell Biology, Academic Centre for Dentistry Amsterdam (ACTA), University of Amsterdam and VU University Amsterdam, Amsterdam, The Netherlands

3 Department of Oral Kinesiology, Academic Centre for Dentistry Amsterdam (ACTA), University of Amsterdam and VU University Amsterdam, Amsterdam, The Netherlands

4 Department of Oral Kinesiology, Academic Centre for Dentistry Amsterdam (ACTA), University of Amsterdam and VU University Amsterdam, Amsterdam, The Netherlands 
improve existing treatment protocols of degenerative joint disease of the TMJ, and also to better understand the regenerative pathway of this particular type of cartilage.

Keywords Mechanical loading · Fibrocartilage · Temporomandibular joint · Cartilage degradation

\section{Introduction}

The temporomandibular joint (TMJ) is covered by fibrocartilage, and its turnover depends on a balance between synthesis and degradation of the extracellular matrix (ECM). Synthesis of the ECM involves the production of collagen fibers, proteoglycans, and aggrecans, and its degradation is caused by the action of enzymes such as aggrecanases and matrix metalloproteinases (MMPs). An important mechanism responsible for the regulation of ECM turnover in the TMJ is mechanical loading [1, 2].
Two categories of mechanical loading can be discerned in the TMJ. The first is static loading, which occurs during teeth clenching, jaw bracing, and activities like swallowing. The second is dynamic loading, which occurs during tooth grinding, jaw thrusting, talking, and chewing. Bone and cartilage are responsible for transmitting and absorbing this mechanical loading [3, 4].

As cartilage is avascular, it needs to receive nutrients from the synovial fluid. This occurs by diffusion due to the movement of the fluid in and out of the cartilage matrix. This movement is caused by the cyclic mechanical loading of the joints (pumping). Pumping may also influence the diffusion of some solutes, such as growth factors, hormones, enzymes and their inhibitors, and cytokines towards the cells. In addition, cyclic mechanical loading helps the drainage of acidic waste materials, such as lactate and $\mathrm{CO}_{2}$ [5]. Future in vitro or finite element studies could elucidate the mechanism of activation of chondrocytes (i.e., direct transduction of mechanical signals to the chondrocytes vs. activation of chondrocytes by facilitated diffusion) in response to TMJ cartilage loading.
Fig. 1 PRISMA flow chart: The flow describes the information through the different phases of a systematic review. It maps out the number of records identified, those included and excluded, and the reasons for exclusions
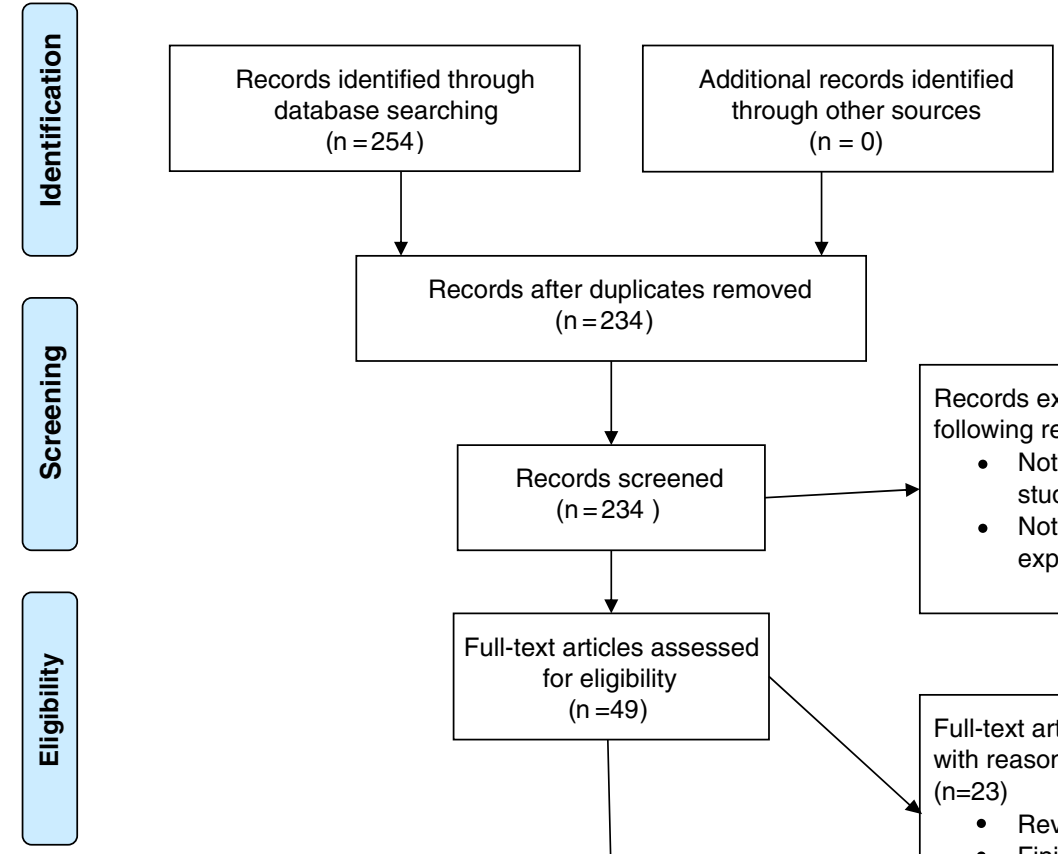
$(n=254)$
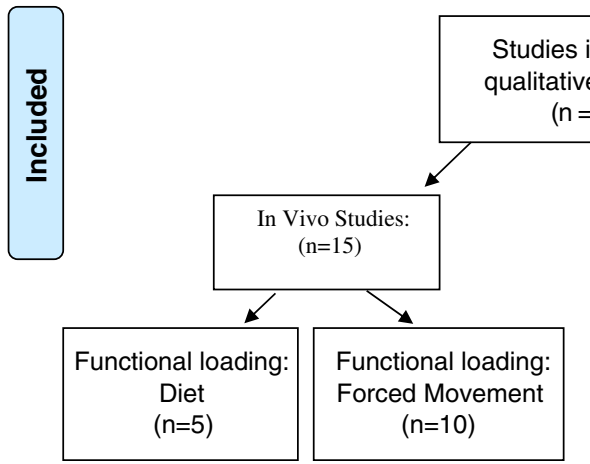

Studies included in ative synthesis $(n=26)$

Full-text articles assessed for eligibility $(n=49)$

Full-text articles excluded with reasons:

$(n=23)$

- Reviews,

- Finite element,

- Risk of Bias ollowing reasons: $(n=185)$

Not in vivo or in vitro studies,

- Not mechanical loading experiments;

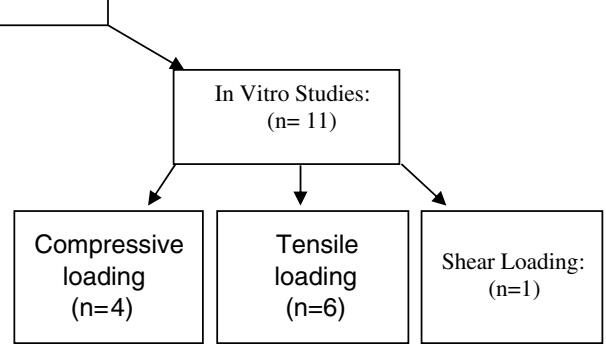


Thus, stimuli induced by mechanical loading can be highly beneficial for the maintenance and integrity of articular cartilage, as well as the development of the mandibular condyle [6].

While moderate dynamic loading is known to maintain the integrity of articular tissue during turnover and growth (anabolic effect), overloading can induce cartilage degradation (catabolic effect) [7]. It is not clear yet how these different loading intensities affect the TMJ cartilage, because in contrast with most synovial joints, which are covered by hyaline cartilage, the TMJ is covered by fibrocartilage. The collagen fibers contained in this TMJ fibrocartilage may provide some additional resistance against mechanical loading.

Knowledge about the way TMJ fibrocartilage responds to different types and intensities of mechanical loading is important to improve existing treatment protocols of degenerative joint disease (DJD) of the TMJ [8], and also to better understand the regenerative pathway of this particular type of cartilage. Therefore, we conducted this systematic review to find out how the TMJ fibrocartilage is affected by different modes of mechanical loading.

\section{Materials and methods}

A review protocol was developed based on the Preferred Reporting Items for Systematic Reviews and Meta-Analysis (PRISMA) statement (www.prisma-statement.org). Embase. com, PubMed, and ISI/Web of Science were searched (by BFB and JCFK) from inception up to September 20th 2016 (see Supplementary information/Search strategy).

The following terms were used (including synonyms and closely related words) as index terms or free-text words: "bite force" or "shear stress" or "mechanical loading" and "cartilage" and "temporomandibular joint." The full search strategies for all the databases can be found in the Supplementary Information. Duplicate articles were excluded. Articles written in English were accepted.

The articles were selected by two independent authors (BFB and VE), following a priori formulated inclusion criteria (viz., in vivo and in vitro studies, mechanical loading experiments on TMJ, and the response of the TMJ). After a subsequent analysis of confounding factors and quality of the research design, papers with sufficient quality were finally selected for this review.

\section{Results}

\section{Literature identification}

With the above-described literature search strategy, 254 records were identified. The complete inclusion process is shown in Fig. 1. No additional records were identified through other sources. After removal of duplicates, 234 records were screened by assessing eligibility criteria for inclusion.

Forty-nine articles were selected after the eligibility inclusion and exclusion criteria for a full-text assessment. Of those, 23 articles were excluded for the following reasons: reviews of experimental studies or abstracts $(n=12)$, a finite element study $(n=1)$, or presence of risk of bias $(n=10)$ (Tables 1 and 2).

Twenty-six experimental studies were included in this systematic review: 15 were in vivo studies, of which 5 were dealing with changes in the hardness of diet and 10 were focusing on forced movement, and 11 were in vitro studies, of which 4 were dealing with compressive loading on the chondrocytes, 6 with tensile loading, and 1 with shear loading.

\section{Main findings}

A wide variation of studies was included in this review. To enable sensible comparison of the results, several groups of studies were distinguished.

In the in vivo studies, different food consistencies, forced jaw movements (by the application of intraoral devices to restrict the jaw position or motion), or surgical intervention (e.g., osteotomies) were used to cause an alteration of the habitual mechanical loading, resulting in a change of the amplitude and/or direction of the TMJ loading. The effect of the

Table 1 Risk of bias (exclusion criteria)

\begin{tabular}{|c|c|}
\hline Study & Reason for exclusion \\
\hline $\begin{array}{l}\text { Pirttiniemi et al. year } \\
\text { (1996) }\end{array}$ & Lack of proper controls ${ }^{\mathrm{a}}$ \\
\hline $\begin{array}{l}\text { Herring et al. year } \\
\text { (2002) }\end{array}$ & $\begin{array}{l}\text { Possible local differences in loading were not } \\
\text { analyzed. The study can only be used to } \\
\text { indicate the site of proliferation but does not } \\
\text { show the effect of loading on proliferation. }\end{array}$ \\
\hline $\begin{array}{l}\text { Wattanachai et al. } \\
\text { (2009) }\end{array}$ & Lack of proper controls ${ }^{\mathrm{b}}$ \\
\hline Fujimura et al. (2005) & Lack of proper controls ${ }^{\mathrm{b}}$ \\
\hline Pirttiniemi et al. (2004) & Lack of proper controls ${ }^{\mathrm{a}}$ \\
\hline Tuominen et al. (1996) & Lack of proper controls ${ }^{\mathrm{a}}$ \\
\hline Magara et al. (2012) & Lack of proper controls ${ }^{\mathrm{b}}$ \\
\hline Wen et al. (2016) & Lack of proper controls ${ }^{\mathrm{d}}$ \\
\hline Henderson et al. (2015) & Lack of proper controls ${ }^{\mathrm{v}}$ \\
\hline Lin. H et al. (2009) & Lack of proper controls ${ }^{2}$ \\
\hline
\end{tabular}

${ }^{\mathrm{a}}$ The intervention should have been applied to both diet groups: soft and hard diet

${ }^{\mathrm{b}} \mathrm{A}$ sham-operated group should have been added as control

${ }^{\mathrm{c}}$ Unilateral splint could affect the non-loaded joint; a control without splint should have been added

${ }^{\mathrm{d}}$ Lack of a control group with an injection of salubrinal but without loading 


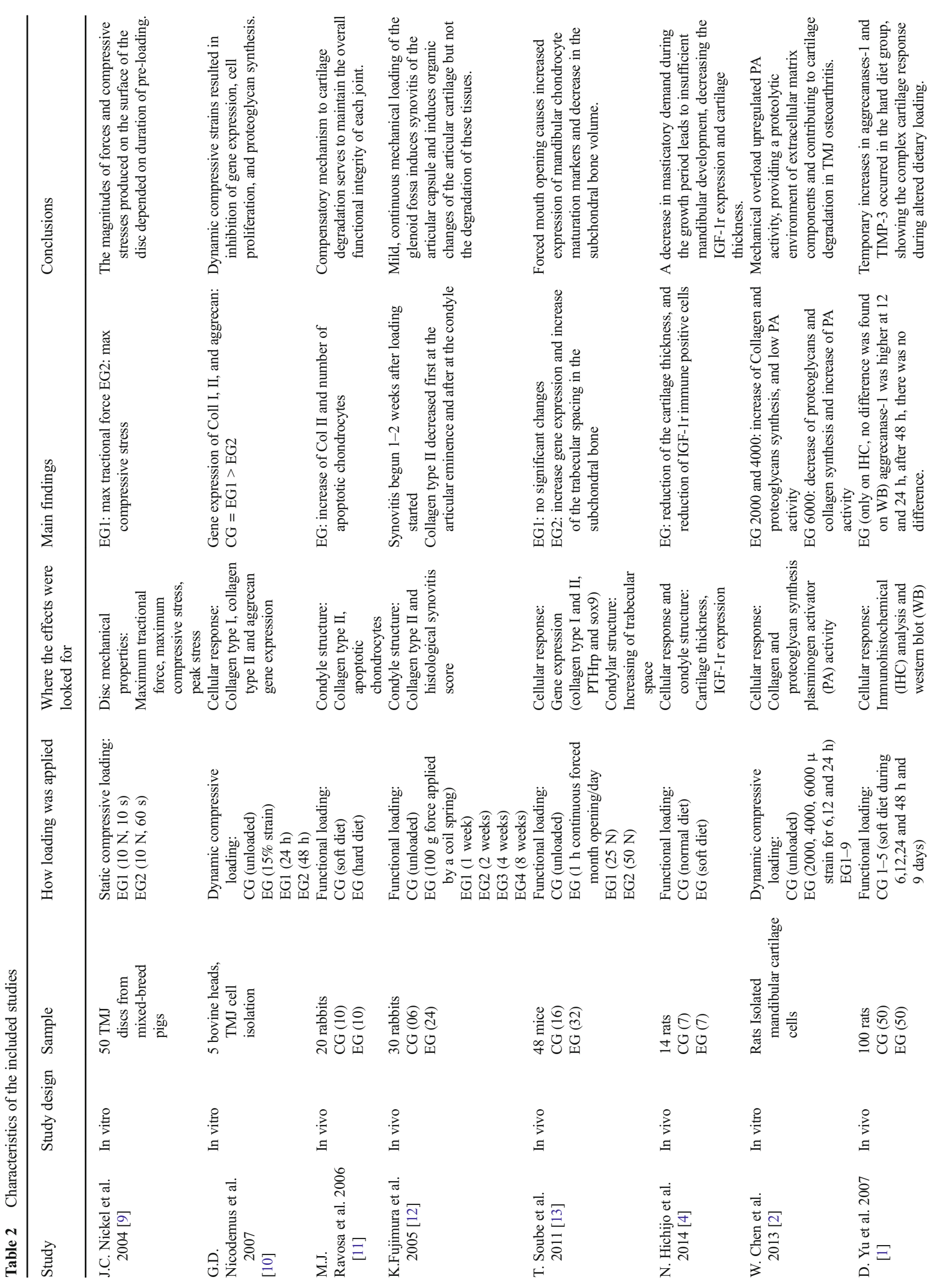




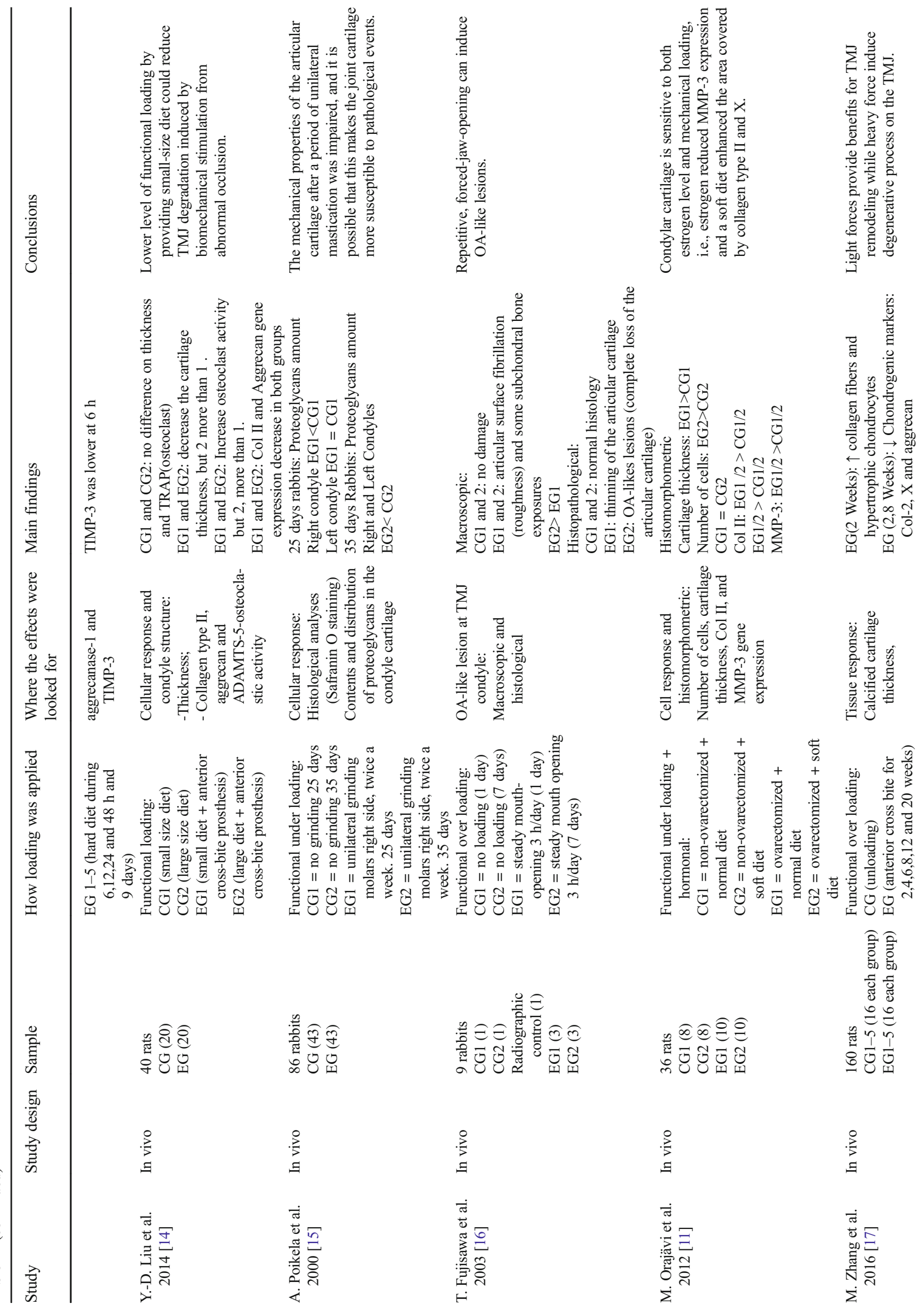




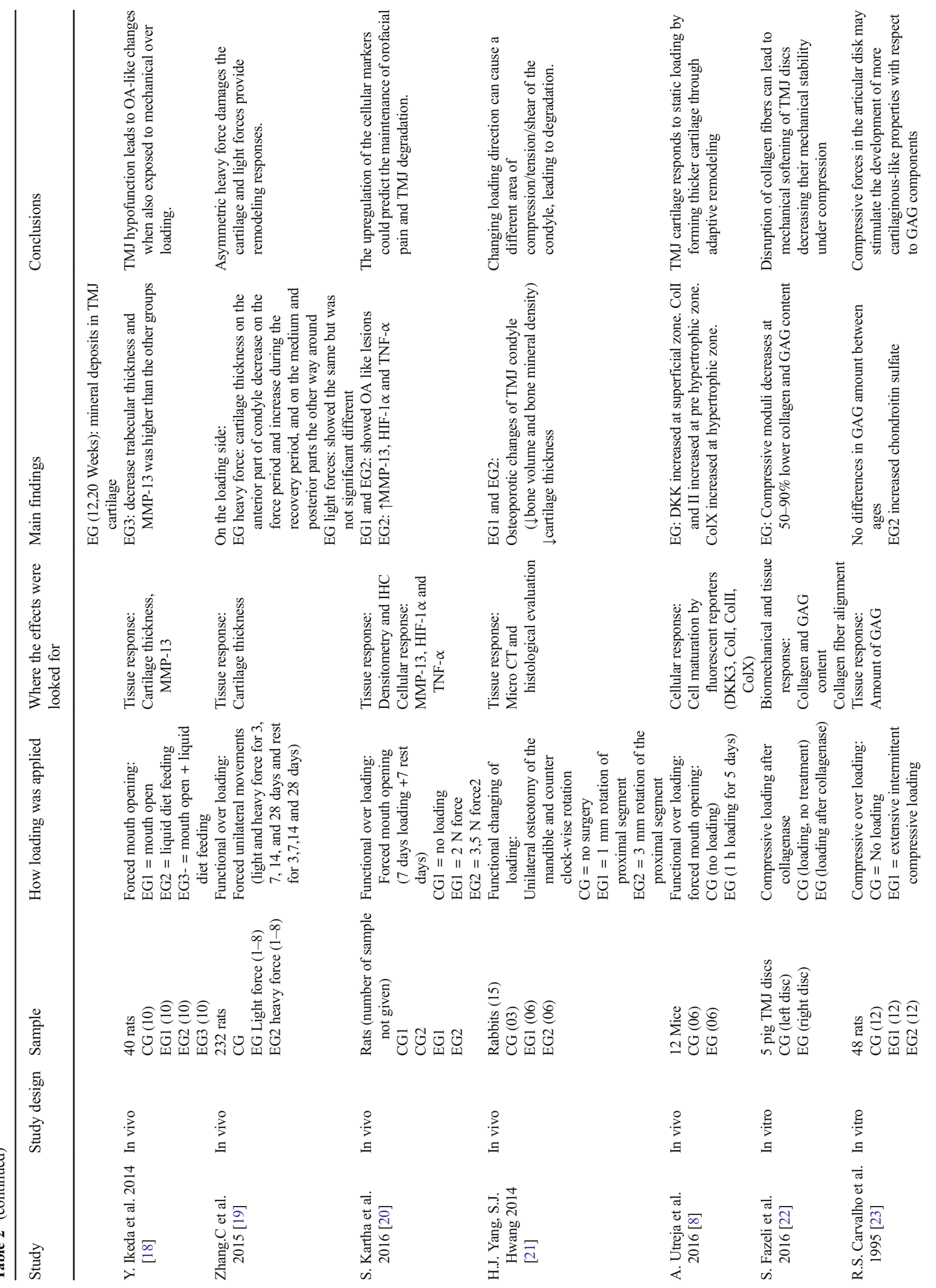




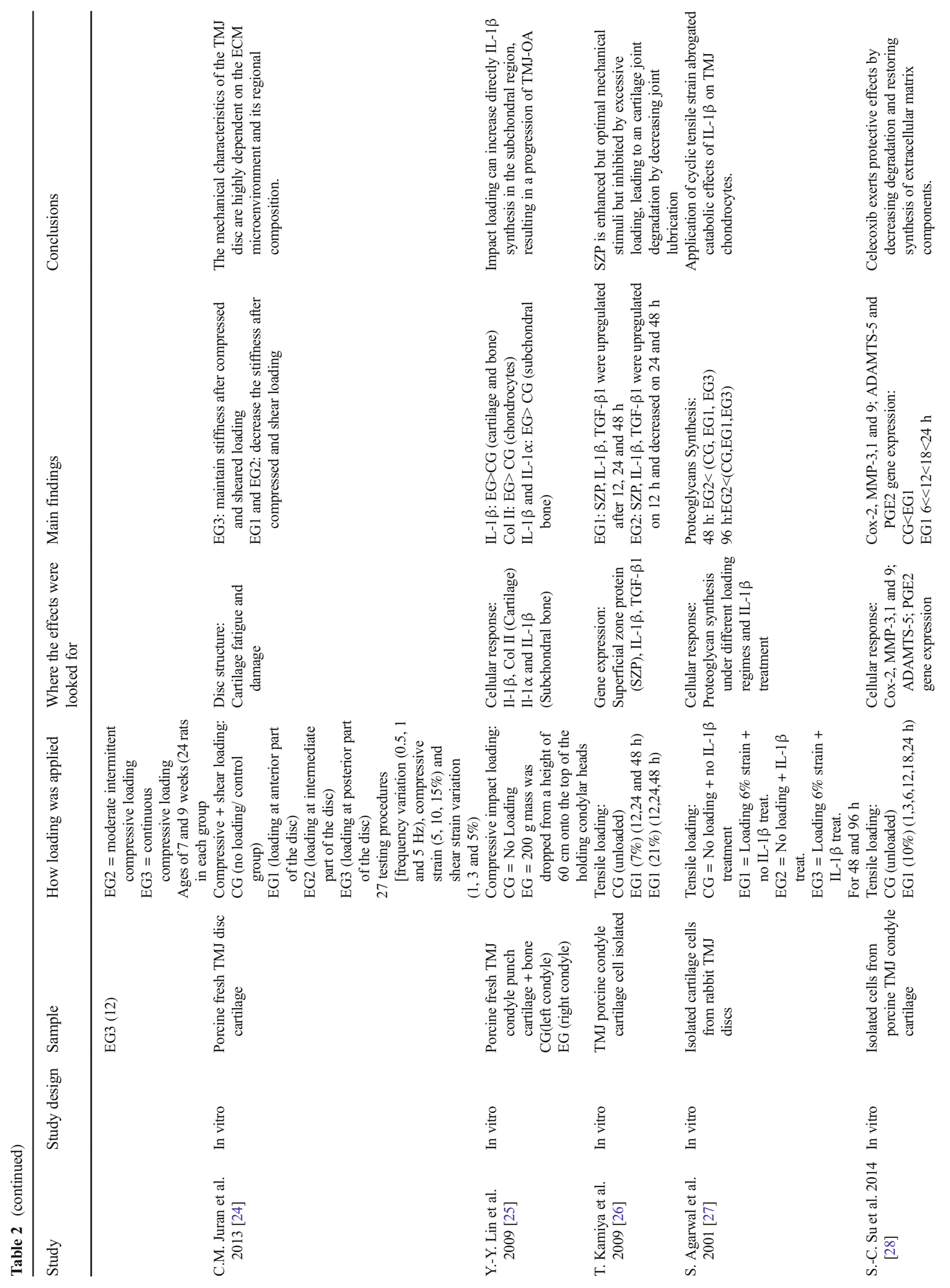


different modes of mechanical loading was analyzed using several parameters. The response of the cartilage was assessed by analyzing either the anatomical structure (DJD like lesions) or the changes of the cellular response using microscopy and/ or assessment of gene expression by polymerase chain reaction PCR.

In the in vitro studies, two types of studies can be identified. In the first type, chondrocytes were isolated from the cartilage and seeded on plates. These cells were then exposed to mechanical forces (compression, tensile, or shear). The response of these cells was determined by analyzing levels of gene expression by PCR. In the second type of studies, fresh pig TMJ discs were exposed to mechanical loading by means of compression, and the outcome measures were quantified as histological changes and alterations of the biomechanics properties of the disc.

\section{Discussion}

\section{Strengths and limitations}

This article aimed to identify the way in which different frequencies and magnitudes of mechanical loading can affect the fibrocartilage of the TMJ. Clear inclusion and exclusion criteria were used to select articles that would be suited to answer the aim. However, as the selected articles used different experimental designs, it was not possible to compare all the articles with each other. For this reason, the set of articles was split by type of study (in vivo and in vitro) and by how the loading was applied.

\section{Interpretation of the evidence}

\section{In vivo experiments}

Excessive, repetitive loading can cause soft- and hard-tissue adaptation or degradation. This was shown when continuous static loading, such as forced mouth opening, was applied in vivo. After 1 day of mouth opening, a catabolic effect was noted: cartilage thickness decreased. The cartilage then adapted to this loading and reacted by increasing the synthesis of collagen type II and other elements of the extracellular matrix [16]. After 1 week of forced moth opening, DJD lesions were nevertheless found $[12,13,19]$.

When the same forced mouth opening protocol was applied with different intensities, light forces provided remodeling of the TMJ, while heavy forces induced degeneration and maintained an inflammatory condition $[15,20]$.

In case of abnormal dynamic and static occlusal relationships, such as unilateral chewing and forced anterior crossbite, the outcomes were always catabolic, with a decrease in the level of proteoglycans and collagen type II, and an 
increase in osteoclastic activity in the condyle [11, 14, 17]. It thus seems that functional overloading skews the balance between ECM formation and degradation in the TMJ towards the latter.

When the mechanical loading consisted of differences in diet hardness, a hard diet, leading to sufficient joint loading, induced an increase in the amount of collagen type II and chondrocyte maturation, thus indicating growth. A soft diet, resulting in a reduced joint loading, reduced cartilage thickness as well as the number of IGF-1 receptor positive cells, indicating reduced growth activity. These results support the importance of mechanical loading (such as chewing) as an essential stimulus to increase mandibular growth [4, 18]. TMJ loading through a hard diet was even able to increase collagen and aggrecan production and cartilage thickness when mechanical overloading was induced through forced mouth opening, thereby preventing cartilage degradation. The hypo function of the TMJ leads to DJD-like lesions [21].

Changes on the direction of the mechanical loading and condyle position after oblique vertical body osteotomy of the mandible and counterclockwise rotation, the same procedure used in Class II orthognathic surgery, induced idiopathic condylar resorption, a kind of DJD. This probably occurred because the trabecular bone patterns reflect the functional loading patterns during the growth period, and this change of condyle position and loading direction exposes an area that is less dense which could decrease the biomechanical properties needed to handle this loading [30].

Apart from loading, hormones may have an effect on the TMJ cartilage. Estrogen seems to inhibit the maturation of the chondrocytes and in cases in which a soft diet loading was applied and was expected to decrease cartilage thickness, such a catabolic effect was partially prevented by the lack of estrogen [25].

\section{In vitro experiments}

In vitro experiments showed that different types of loading regimes, such as tension, compression, and shear, had different effects on the TMJ cartilage chondrocytes when applied at low, moderate, or high intensity. At high intensity, tension and compression both caused a catabolic effect on the chondrocytes by reducing gene expression of the extracellular matrix components and increasing IL1- $\beta$ production [26]. Unlike high intensity, low and moderate dynamic compression had an anabolic effect on the chondrocytes, increasing the expression of collagen type I and II and aggrecans [10]. These effects are timedependent, as Nicodemus et al. [23] showed after application of dynamic compressive overloading. During the first $24 \mathrm{~h}$, the gene expression of collagen type I and II and aggrecan increased, showing an adaptation behavior. After $48 \mathrm{~h}$, the gene expression decreased to a level under the control levels, which demonstrates a catabolic effect of prolonged loading.
The reaction of TMJ disc-derived cells to compression is also time-dependent. When compression was applied for a short period and with longer intervals between cycles, fibrocartilage had more time to recover and return to the initial stage $[9,22]$. This capacity to recover is changed when the collagen fiber network is disrupted, i.e., after a collagenase treatment as shown in fresh porcine discs [24]. Such a situation can occur in vivo in cases of intra-articular inflammation where cytokines stimulate degradation of collagen fibers. When shear movements were applied, the different parts of the TMJ disc reacted differently. The posterior zone was more resistant, with better biomechanical properties, and showed less deformation during loading than the anterior and intermediate zones of the disc [27].

In addition to a catabolic effect, cyclic tensile strain can also protect the cartilage from the effects of inflammation, e.g., suppressing the catabolic effect of TNF- $\alpha$ by downregulating the expression of MMPs by TNF- $\alpha$-treated chondrocytes [28, 29, 31]. As well as cyclic tensile strain, celecoxib has a protective effect by decreasing degradation and restoring synthesis of ECM in inflamed cartilage [32].

\section{Recommendations}

More in vivo and in vitro studies in each type of study design are required to clarify how fibrocartilage reacts to different types of mechanical loading. In this regard, we would like to stress the importance of physical measurements of actual loading conditions in the tissues, as these can be quite different from what is assumed. For example, Rafferty et al. demonstrated that during mandibular distraction in minipigs, the increased cartilage thickness on the distraction side was associated with reduced rather than increased loading [33].

In addition, studies are needed to assess how mechanical loading could be incorporated in new protocols for the treatment of DJD, for example by including physiotherapy (e.g., cyclic loading). In vivo studies on the efficacy of orthognathic surgery on the TMJ would be important to predict side effects and to prevent idiopathic condyle resorption in patients.

The mechanical loading described in the included in vivo studies only includes diet and overloading by forced mouth opening and other artificial interventions, but it would be interesting to include other kinds of loading as well, mimicking clenching and grinding, and to assess how the TMJ reacts to these different intensities and frequencies of mechanical loading.

\section{Conclusion}

Based on the studies included in this review, we could conclude that dynamic mechanical loading is an important stimulus for mandibular growth and for the homeostasis of TMJ 
cartilage. When this loading is applied at a low intensity, it protects inflamed cartilage by effectively antagonizing IL-1 $\beta$. However, frequent overloading induces accelerated cell death and increased cartilage degradation.

\section{Compliance with ethical standards}

Conflicts of interest The authors declare that they have no conflict of interest.

Funding This research did not receive any specific grant from funding agencies in the public, commercial, or not-for-profit sectors.

Ethical approval This article does not contain any studies with human participants or animals performed by any of the authors.

Informed consent For this type of study, formal consent is not required

Open Access This article is distributed under the terms of the Creative Commons Attribution 4.0 International License (http:// creativecommons.org/licenses/by/4.0/), which permits unrestricted use, distribution, and reproduction in any medium, provided you give appropriate credit to the original author(s) and the source, provide a link to the Creative Commons license, and indicate if changes were made.

\section{References}

1. Yu D, Tiilikainen P, Raustia A, Pirttiniemi P (2007) Dietary loading and aggrecanase-1/TIMP-3 expression in rat mandibular condylar cartilage. J Orofac Pain 21:232-238

2. Chen W, Tang Y, Zheng M, Jiang J, Zhu G, Liang X, Li M (2013) Regulation of plasminogen activator activity and expression by cyclic mechanical stress in rat mandibular condylar chondrocytes. Mol Med Rep 8:1155-1162

3. Schlaak JF, Pfers I, Meyer Zum Büschenfelde KH, MärkerHermann E (1996) Different cytokine profiles in the synovial fluid of patients with osteoarthritis, reumatoid arthritis and seronegative spondylarthropathies. Clin Exp Rheumatol 14:155-162

4. Hichijo N, Kawai N, Mori H, Sano R, Ohnuki Y, Okumura S, Langenbach GEJ, Tanaka E (2014) Effects of the masticatory demand on the rat mandibular development. J Oral Rehabil 41:581587

5. O'Hara BP, Urban JP, Maroudas A (1990) Influence of cyclic loading on the nutrition of articular cartilage. Ann Rheum Dis 49:536539

6. Copray JVM, Dibbets JMH, Kantoma T (1988) The role of condylar cartilage in the development of the temporomandibular joint. Angle Orthodon 58:369-380

7. Sun HB (2010) Mechanical loading, cartilage degradation, and arthritis. Ann N Y Acad Sci 1211:37-50

8. Schiffman E, Ohrbach R, Truelove E, Look J, Anderson G, Goulet JP, List T, Svensson P, Gonzalez Y, Lobbezoo F, Michelotti A, Brooks SL, Ceusters W, Drangsholt M, Ettlin D, Gaul C, Goldberg L, Haythornthwaite J, Hollender L, Jensen R, John MT, De Laat A, De Leeuw R, Maixner W, van der Meulen M, Murray GM, Nixdorf DR, Palla S, Petersson A, Pionchon P, Smith B, Visscher CM, Zakrzewska J, Dworkin SF (2014) Diagnostic criteria for temporomandibular disorders (DC/TMD) for clinical and research applications: recommendations of the international RDC/ TMD consortium network and orofacial pain special interest group. J Oral Facial Pain Headache 28:6-27
9. Carvalho RS, Yen EHK, Suga DM (1995) Glycosaminoglycan synthesis in the rat articular disk in response to mechanical stress. Am J Orthod Dentofac Orthop 107:401-410

10. Kamiya T, Tanimoto T, Tanne Y, Lin YY, Kunimatsu R, Yoshioka M, Tanaka N, Tanaka E, Tanne K (2010) Effects of mechanical stimuli on the synthesis of superficial zone protein in chondrocytes. J Biomed Mater Res A 92:801-805

11. Zhang M, Wang H, Zhang J, Zhang H, Yang H, Wan X, Jing L, Lu L, Liu X, Yu S, Chang X, Wang M (2016) Unilateral anterior crossbite induces aberrant mineral deposition in degenerative temporomandibular cartilage in rats. Osteoarthr Cartil 24:921-931

12. Soube T, Yeh W-C, Chhibber A, Ultreja A, Diaz-Doran V, Adams D, Wadhwa S (2011) Murine TMJ loading causes increased proliferation and chondrocyte maturation. J Dent Res 90:512-516

13. Fujisawa T, Kuboki T, Kasai T, Sonoyama W, Kojima S, Uehara J, Komori C, Yatani H, Hattori T, Takigawa M (2003) A repetitive, steady mouth opening induced an osteoarthritis-like lesion in the rabbit temporomandibular joint. J Dent Res 82:731-735

14. Poikela A, Kantomaa T, Perttiniemi P, Tuukkanen J, Pietilä K (2000) Unilateral masticatory funcition chnages the proteoglycan content of mandibular condylar cartilage in rabbit. Cells Tissues Organs 167:49-57

15. Kartha S, Zhou T, Granquist EJ, Winkelstein BA (2016) Development of a rat model of mechanically induced tunable pain and associated temporomandibular joint responses. J Oral Maxillofac Surg 74:54.e1-54.e10

16. Utreja A, Yadav S, Villa MM, Li Y, Jiang X, Nanda R, Rowe DW (2016) Cell and matrix response of temporomandibular cartilage to mechanical loading. Osteoarthr Cartil 24:335-344

17. Liu Y-D, Liao L-F, Zhang H-Y, Lu L, Jiao K, Zhang M, Zhang J, He J-J, Wu Y-P, Chen D, Wang M-Q (2014) Reducing dietary loading decreases mouse temporomandibular joint degradation induced by anterior crossbite prosthesis. Osteoarthr Cartil 22:302-312

18. Ravosa MJ, Kunwar R, Stock SR, Stack MS (2007) Pushing to the limit: masticatory stress and adaptative plasticity in mammalian craniomandibular joints. J Exp Biol 210:628-641

19. Fujimura K, Kobayashi S, Susuki T, Segami N (2005) Histologic evaluation of temporomandibular arthritis induced by mild mechanical loading in rabbits. J Oral Pathol Med 34:157-163

20. Zhang C, Xu Y, Cheng Y, Wu T, Li H (2015) Effect of asymmetric force on the condylar cartilage, subchondral bone and collagens in the temporomandibular joints. Arch Oral Biol 60:650-663

21. Ikeda Y, Yonemitsu I, Takei M, Shibata S, Ono T (2014) Mechanical loading leads to osteoarthritis-like changes in the hypofunctional temporomandibular joint in rats. Arch Oral Biol 59:1368-1376

22. Nickel JC, Iwasaki LR, Beatty MW, Marx DB (2004) Laboratory stresses and tractional forces on the TMJ disc surface. J Dent Res 83:650-654

23. Nicodemus GD, Villanueva I, Bryant SJ (2007) Mechanical stimulation of TMJ condylar chondrocytes in PEG hydrogels. J Biomed Mater Res A 83:323-331

24. Fazaeli S, Ghazanfari S, Everts V, Smit TH, Koolstra JH (2016) The contribution of collagen fibers to the mechanical compressive properties of the temporomandibular joint disc. Osteoarthr Cartil 24: 1292-1301

25. Orajärvi M, Puijola E, Yu S-B, Liu X, Tiilikainen P, Wang M, Raustia A, Perttiniemi P (2012) Effect of estrogen and dietary loading on condylar cartilage. J Orofac Pain 26:328-336

26. Lin Y-Y, Tanaka N, Ohkuma S, Kamiya T, Kunimatsu R, Huang YC, Yoshioka M, Mitsuyoshi T, Tanne Y, Tanimoto K, Tanaka E, Tanne K (2009) The mandibular cartilage metabolism is altered by damaged subchondral bone from traumatic impact loading. Ann Biomed Eng 37:1358-1367 
27. Juran CM, Dolwick MF, McFetrige PS (2013) Shear mechanics of the TMJ disc: relationship to common clinical observations. J Dent Res 92:193-198

28. Tabeian H, Bakker AD, Betti BF, Lobbezoo F, Everts V, de Vries TJ (2016) Cyclic tensile strain reduces TNF- $\alpha$ induced expression of MMP-13 by condylar temporomandibular joint cells. J Cell Physiol 232:1287-1294

29. Deschner J, Rath-Deschner B, Agarwal S (2006) Regulation of matrix metalloproteinase expression by dynamic tensile strain in rat fibro chondrocytes. Osteoarthr Cartil 14:264-272

30. Yang HJ, Hwang SJ (2014) Osseous alterations in the condylar head after unilateral surgical directional change in rabbit mandibular condyles: preliminary study. J Craniomaxilofacial Surg 42: 1632-1638
31. Agarwal S, Long P, Gassner R, Piesco NP, Buckley MJ (2001) Cyclic tensile strain suppresses catabolic effects of interleukin- $1 \beta$ in fibrochondrocytes from the temporomandibular joint. Arthritis Reum 44:608-617

32. Su S-C, Tanimoto K, Tanne Y, Kunimatsu R, Hirose N, Mitsuyoshi T, Okamoto Y (2014) Celocoxib exerts protective effects on extracellular matrix metabolism of mandibular condylar chondrocytes under excessive mechanical stress. Osteoarthr Cartil 22:845-851

33. Rafferty KL, Sun Z, Egbert M, Bakko DW, Herring SW (2007) Changes in growth and morphology of the condyle following mandibular distraction in minipigs: overloading or underloading. Arch Oral Biol 52:96976 